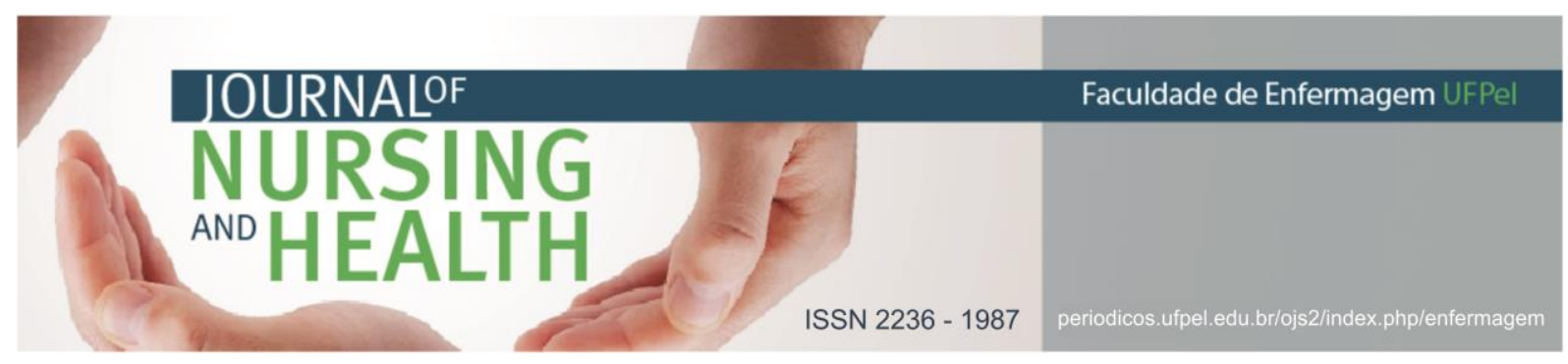

ARTIGO DE REVISÃO

\title{
Ômega-3 na frequência de micronúcleos em células da mucosa bucal em pacientes obesos
}

\section{Omega-3 in the frequency of micronuclei in oral mucosa cells in obese patients}

\author{
Omega-3 en la frecuencia de micronúcleos en las células bucales en pacientes obesos \\ Rameiro, Thyago Francisco Andrade ${ }^{1}$; Campelo, Rômulo Costa²; Batista, Nelson Jorge Carvalho³
}

Como citar este artigo: Rameiro TFA, Campelo RC, Batista NJC. Ômega-3 na frequência de micronúcleos em células da mucosa bucal em pacientes obesos. J. nurs. health. 2020;10(n.esp.):e20104009

\section{RESUMO}

Objetivo: analisar na produção científica sobre o efeito do ômega-3 na determinação da frequência basal de micronúcleos nas células da mucosa oral em pacientes com obesidade. Método: revisão integrativa de publicações dos últimos 10 anos, realizada de fevereiro a junho de 2017, por meio de Biblioteca Virtual de Saúde, Medical Literature Analysis and Retrieval System, Literatura Latinoamericana e do Caribe em Ciências da Saúde e PubMed Central. Resultados: obteve-se 11 publicações, que de modo geral, tratam da ação do ômega- 3 na frequência basal de micronúcleos em células da mucosa oral em pacientes com obesidade e do consumo de ômega-3 como forma de redução da incidência de doenças cardiovasculares e câncer. Conclusões: observou-se à importância do ômega-3 na redução de micronúcleos em células da mucosa oral em pacientes com obesidade, com isso é possível aumentar a eficácia da quimioterapia.

Descritores: Ácidos graxos ômega-3; Mucosa bucal; Testes para micronúcleos

\begin{abstract}
Objective: to analyze in scientific production about the effect of omega 3 in determining the basal frequency of micronuclei in oral mucosa cells in patients with obesity. Method: integrative review of publications from the last 10 years, carried out from February to June 2017, through the Virtual Health Library, Medical Literature Analysis and Retrieval System, Latin American and Caribbean Literature in Health Sciences and PubMed Central. Results: 11 publications were obtained, which deal with the action of omega 3 on the basal frequency of micronuclei in oral mucosa cells in patients with obesity and consumption of omega 3 as a way of reducing the incidence of cardiovascular diseases and cancer. Conclusions: it was observed the importance of omega 3 in the reduction of micronuclei in cells of the oral mucosa in patients with obesity, thus it is possible to increase the effectiveness of chemotherapy.
\end{abstract}

Descriptors: Fatty acids, omega-3; Mouth mucosa; Micronucleus tests

1 Nutricionista. Centro Universitário Santo Agostinho (UNIFSA). Piauí (PI), Brasil. E-mail: julianab48@gmail.com http://orcid.org/0000-0003-3230-4167

2 Enfermeiro. Instituto de Medicina Integral Prof. Fernando Figueira (IMIP). Pernambuco (PE), Brasil. E-mail: romulocam14@gmail.com http://orcid.org/0000-0002-8812-3915

3 Biólogo. Doutor em Biologia Celular Molecular Aplicada à Saúde. Centro Universitário Santo Agostinho (UNIFSA). Piauí (PI), Brasil. E-mail: nelsonjcb@hotmail.com http://orcid.org/0000-0001-8326-1510 


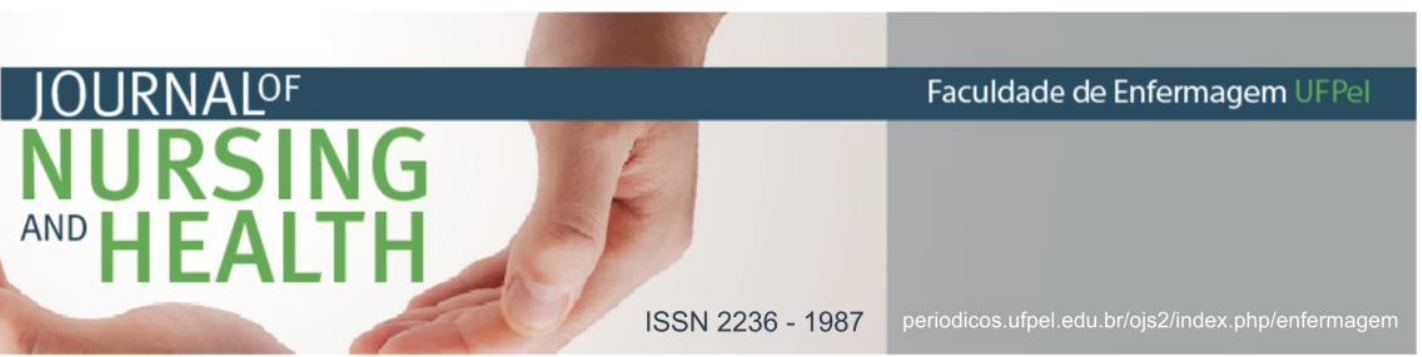

RESUMEN

Objetivo: analizar en la producción científica sobre el efecto de omega-3 en la determinación de la frecuencia basal de micronúcleos en células de la mucosa oral en pacientes con obesidad. Método: revisión integradora de publicaciones de los últimos 10 años, realizada de febrero a junio de 2017, a través de la Biblioteca Virtual en Salud, Medical Literature Analysis and Retrieval System, Literatura Latinoamericana y del Caribe en Ciencias de la Salud y PubMed. Resultados: se obtuvieron 11 publicaciones, sobre la acción de omega-3 en la frecuencia basal de micronúcleos en células de la mucosa oral en pacientes con obesidad y consumo de omega-3 como forma de reducir la incidencia de enfermedades cardiovasculares y cáncer. Conclusiones: se observó la importancia de omega 3 en la reducción de micronúcleos en células de la mucosa oral en pacientes con obesidad, con esto es posible aumentar la efectividad de la quimioterapia.

Descriptores: Ácidos grasos omega-3; Mucosa bucal; Pruebas de micronúcleos

\section{INTRODUÇÃO}

A obesidade, considerada em séculos passados como símbolo de fartura, saúde e beleza, é uma doença crônica multifatorial caracterizada pelo excesso de gordura corporal decorrente da hiperplasia e/ou hipertrofia dos adipócitos.

Na relação obesidade e nutrição o elemento ômega-3 pode ser importante. O ômega-3 é classificado como Ácido Graxo (AG) poli-insaturado de cadeia longa por ter 14 a 22 átomos de carbono e recebe a denominação ômega-3 por conter a primeira dupla ligação no carbono, a partir do radical metil. 1

Vários alimentos têm contribuído para o surgimento do câncer, em especial o câncer de mama, intestino grosso, reto, próstata, esôfago e estômago. Entre os alimentos que aumentam o risco para desenvolver o câncer, estão: as frituras, carnes com gorduras aparentes, carnes processadas como salsichas, linguiças defumadas, presuntos, salames, bacon, toucinho, carnes enlatadas. ${ }^{2}$

A capacidade carcinogênica dos alimentos industrializados está relacionada com a ação dos componentes alimentares no organismo. É conveniente a contínua atenção com os elementos químicos sintetizados, para que estes não estimulem o câncer, sendo os agrotóxicos um dos principais ingredientes adicionados na maioria dos alimentos da produção agrícola. Em um estudo, demonstra-se que boa parte desses corantes adicionados nos refrigerantes podem ser tóxicos às células e causar mutações celulares. ${ }^{3}$

0 teste de Micronúcleos ( $M N)$ em células bucais esfoliadas é um método minimamente invasivo para causar dano ao Ácido Desoxirribonucleico (DNA), instabilidade cromossômica, morte celular e o potencial regenerativo do tecido da mucosa bucal humana. As células com micronúcleos são aquelas que se caracterizam pela presença de um núcleo principal e outras estruturas nucleares menores, denominadas micronúcleos. ${ }^{4}$

O MN é um método eficiente, de baixo investimento e um modo confiável para mensurar a instabilidade genética em grandes populações. Assim, amostras de células 
da mucosa bucal têm sido utilizada para investigar o impacto da nutrição e do ômega-3 sobre o dano ao DNA e morte celular. ${ }^{5}$

O MN é um pequeno núcleo adicional que se forma a partir de mutações cromossômicas ou fragmentadas durante a divisão celular. A quebra ou anomalia estrutural assimétrica de um cromossomo pode estar relacionada ao não reparo ou lesões de DNA ou má expressão de cromossomo, devido ao seu mau funcionamento mitótico. Com o notável aumento da frequência de MN em células esfoliadas, observa-se uma grande variedade de condições, tal qual à exposição aos pesticidas, à radioterapia, ao tabagismo, ao estresse oxidativo e aos defeitos genéticos no checkpoint do ciclo celular ou reparo do DNA. ${ }^{6}$

Por essa razão, o presente trabalho propõe a seguinte indagação: como o ômega-3 pode influenciar na redução de micronúcleos em células basais da mucosa oral em pacientes com obesidade? E objetiva analisar na produção científica do efeito do ômega-3 na determinação da frequência basal de micronúcleos nas células da mucosa oral em pacientes com obesidade.

\section{MATERIAS E MÉTODOS}

0 presente estudo trata-se de uma revisão integrativa de literatura realizada no período de fevereiro a junho de 2017, por meio da plataforma da Biblioteca Virtual de Saúde (BVS) foi acessada a Literatura Latinoamericana e do Caribe em Ciências da Saúde (LILACS) e na base de dados Medical Literature Analysis and Retrieval System (MEDLINE), o Public Publisher MEDLINE (PubMed). Utilizaram-se como descritores (DeCS): Ácidos graxos Ômega-3, obesidade, mucosa oral e testes para micronúcleos e outros cadastrados no Medical Subject Headings (MeSH): Omega-3 fatty acids, obesity, oral mucosa and tests for micronuclei. Para a busca adotou-se a estratégia PICO $(\mathrm{P}=$ população ou problema, $\mathrm{I}=$ intervenção, $\mathrm{C}=$ comparação, $\mathrm{O}=$ resultado), a partir da qual foram elaboradas equações de busca para coleta dos artigos (Quadro 1).

Quadro 1: Estratégia PICO para busca dos dados. Teresina, 2017

\begin{tabular}{|c|c|c|c|c|}
\hline Acrônimo & \multicolumn{2}{|c|}{ Descritor/palavras-chave } & \multicolumn{2}{|c|}{ Equação de busca } \\
\hline \multirow[b]{2}{*}{$\mathbf{P}$} & BVS & PubMed & BVS & PubMed \\
\hline & $\begin{array}{l}\text { Obesidade, Mucosa } \\
\text { Bucal }\end{array}$ & $\begin{array}{l}\text { Obesity, Buccal } \\
\text { Mucosa }\end{array}$ & $\begin{array}{l}\text { (tw: (Obesidade)) AND } \\
\text { (tw: (Mucosa Bucal)) }\end{array}$ & $\begin{array}{l}\text { (Buccal Mucosa) } \\
\text { AND Obesity }\end{array}$ \\
\hline I & $\begin{array}{c}\text { Testes para } \\
\text { Micronúcleo, Ácidos } \\
\text { Graxos Ômega-3 }\end{array}$ & $\begin{array}{l}\text { Tests for } \\
\text { Micronuclei, } \\
\text { Fatty Acids }\end{array}$ & $\begin{array}{l}\text { (tw: (Ácidos Graxos } \\
\text { Ômega-3)) AND } \\
\text { (tw:(Testes para } \\
\text { Micornucleos)) }\end{array}$ & $\begin{array}{l}\text { (Fatty Acids) } \\
\text { AND Tests for } \\
\text { Micronuclei }\end{array}$ \\
\hline C & - & - & - & - \\
\hline 0 & Micronúcleo & Micronuclei & (tw: (Micronucleo)) & Micronuclei \\
\hline $\begin{array}{l}\text { Equação } \\
\text { geral de }\end{array}$ & \multicolumn{4}{|c|}{$\begin{array}{l}\text { BVS = (tw:(Obesidade)) AND (tw:(Mucosa Bucal)) AND (tw:( Testes para } \\
\text { Micronucleos)) AND (tw:(Ácidos Graxos Omega-3)) AND (tw:(Micronucleo)) }\end{array}$} \\
\hline busca & \multicolumn{4}{|c|}{ PubMed $=((($ Fatty Acids) AND Tests for Micronuclei) AND Buccal Mucosa) AND Obesity } \\
\hline
\end{tabular}

Fonte: Dados da Pesquisa, 2017. 
Foram incluídos os artigos em português, inglês e espanhol que: tratavam da ação do ômega-3 na frequência basal de micronúcleos em células da mucosa oral em pacientes com obesidade; foram publicados no período de 2007 a 2017 e estavam descritos na íntegra. Foram excluídos os artigos que não correspondiam ao objeto de estudo. As buscas iniciais encontraram 2.178 e após a filtragem com os critérios de inclusão em exclusão restaram 11 (Tabela 1).

Tabela 1: Distribuição das referências obtidas nas bases de dados consultadas, seguindo os descritores estabelecidos, Teresina, 2017

\begin{tabular}{|c|c|c|c|}
\hline Base de dados & Descritores cruzados & $\begin{array}{l}\text { Referências } \\
\text { obtidas }\end{array}$ & $\begin{array}{l}\text { Referências } \\
\text { selecionadas }\end{array}$ \\
\hline MEDLINE & Ácidos Graxos Ômega-3 AND Obesidade & 419 & 2 \\
\hline MEDLINE & $\begin{array}{c}\text { Ácidos Graxos Ômega-3 AND Testes para } \\
\text { Micronúcleos }\end{array}$ & 1 & 1 \\
\hline MEDLINE & Testes para Micronúcleos AND Mucosa Oral & 228 & 1 \\
\hline LILACS & Ácidos Graxos Ômega-3 AND Obesidade & 5 & 2 \\
\hline LILACS & $\begin{array}{c}\text { Ácidos Graxos Ômega-3 AND Testes para } \\
\text { Micronúcleos }\end{array}$ & 0 & 0 \\
\hline LILACS & Testes para Micronúcleos AND Mucosa Oral & 26 & 1 \\
\hline PubMed & Omega-3 Fatty Acids AND Obesity & 1249 & 2 \\
\hline PubMed & $\begin{array}{c}\text { Omega-3 Fatty Acids AND Tests for } \\
\text { Micronuclei }\end{array}$ & 3 & 1 \\
\hline PubMed & Tests for Micronuclei AND Oral Mucosa & 247 & 1 \\
\hline
\end{tabular}

Fonte: Dados da Pesquisa, 2017.

As publicações foram selecionadas após a leitura minuciosa dos resumos, que tratam da ação do ômega-3 na frequência basal de micronúcleos como objeto de estudo, procedendo em seguida, para a análise e interpretação dos resultados. Os artigos foram lidos e analisados de forma sistematizada.

\section{RESULTADOS}

De modo geral, os estudos selecionados tratam da ação do ômega-3 na frequência basal de micronúcleos em células da mucosa oral em pacientes com obesidade e do consumo de ômega-3 como forma de redução da incidência de doenças cardiovasculares e câncer.

Em relação aos autores e tipo de estudo, observa-se que os autores não publicaram mais de um artigo envolvendo a mesma temática. Os estudos brasileiros apresentaram predominância da abordagem qualiquantitativa, o que não aconteceu com os publicados internacionalmente (Quadro 2).

De acordo com o levantamento realizado nas bases de dados MEDLINE, 


\section{NURSING \\ AND HEALTH}

ISSN 2236 - 1987

LILACS e PubMed, contemplaram-se as produções acerca do consumo de ômega-3 como forma de redução da incidência de doenças cardiovasculares e câncer.

Em relação aos anos de publicação dos estudos, identificou-se que o ano de 2011 teve um maior número de publicações do que os outros anos. Nos anos de 2008,2009 e 2012 não foram encontradas publicações.
Quanto ao periódico, percebeu-se uma quantidade igual de publicações em todas as revistas. Quanto à metodologia, observou-se uma diversidade nos estudos analisados, caracterizando a maior frequência de quali-quantitativo, seguidos pelos de abordagem quantitativo, qualitativo e estudo piloto. Foi possível destacar que cinco artigos estavam disponíveis na língua portuguesa e seis na língua inglesa.

Quadro 2: Distribuição das referências incluídas na revisão integrativa, de acordo com as bases de dados: idioma, área de atuação e tipo de estudo. Teresina, 2017

\begin{tabular}{|c|c|c|c|}
\hline Base de dados & Idioma & Área & Tipo de estudo \\
\hline LILACS & Inglês & Médica & Quali-quantitativo \\
\hline MEDLINE & Inglês & Nutricionista & Qualitativo \\
\hline PubMed & Português & Nutricionista & Quali-quantitativo \\
\hline MEDLINE & Inglês & Nutricionista & Qualitativo \\
\hline LILACS & Inglês & Médica & Quali-quantitativo \\
\hline LILACS & Inglês & Nutricionista & Quantitativo \\
\hline MEDLINE & Inglês & Médica & Quali-quantitativo \\
\hline PubMed & Português & Nutricionista & Quantitativo \\
\hline MEDLINE & Português & Médica & Quali-quantitativo \\
\hline PubMed & Português & Nutricionista & Quali-quantitativo \\
\hline PubMed & Português & Nutricionista & Quali-quantitativo \\
\hline
\end{tabular}

Fonte: Dados da Pesquisa, 2017.

\section{DISCUSSÃO}

Ao analisar os resultados obtidos, pode-se observar que alguns trabalhos possuem linhas de pesquisa comuns entre si, e, considerando seus eixos temáticos, foram criadas duas categorias, a fim de facilitar a discussão entre os autores, que estão descritas a seguir:
Importância do ômega-3 na redução de micronúcleos em células da mucosa oral em pacientes com obesidade

A presença de micronúcleos nas células em proliferação é um sensível marcador de dano cromossômico espontâneo ou induzido. De fato, como um micronúcleo pode ser composto tanto de fragmentos de cromossomos 
acêntricos como pelo atraso de cromossomos inteiros no decorrer da divisão celular, a análise de micronúcleos possibilita a avaliação de eventos clastogênicos (induzidos por quebra da cadeia dupla) e aneuploidogênicos (induzidos por má segregação cromossômica) ao mesmo tempo. $^{7}$

A composição dos fosfolipídios de membranas na forma de AGs é, em parte, influenciada pela composição dos AGs n-3 e n-6 da alimentação. Assim, a composição da gordura alimentar é capaz de determinar inúmeras funções referentes à membrana, como por exemplo: ligação de hormônios e atividades relacionada a enzimas e transportadores. ${ }^{8}$

0 teste de micronúcleos em células bucais esfoliadas é um método minimamente invasivo para analisar o dano ao DNA, instabilidade cromossômica, morte celular e o potencial regenerativo do tecido da mucosa bucal humana. As células com micronúcleos são células que se distingue pela presença de um núcleo principal e outras estruturas nucleares menores, denominadas micronúcleos. ${ }^{7}$

Com a análise citogenética de células esfoliadas em cavidade bucal é possível realizar a coleta de células da mucosa oral ou da borda lateral da língua, pois estes tecidos epiteliais estão em permanente processo de divisão celular, com renovação do tecido pela descamação das células superficiais. As células epiteliais esfoliadas não se dividem mais, mas refletem anormalidades citogenéticas que ocorreram na população de células na camada basal. ${ }^{9}$
Os AGs Eicosapentaenóicos (EPA) e Docosahexaenóicos (DHA) são localizados em peixes de água salgada e, em determinadas sementes, como a linhaça. É possível citar o atum, a sardinha, o salmão e a cavala, uma vez que conforme são mais ricos em gordura, proporcionalmente maior será seu teor de AG n-32. ${ }^{10}$ Os AG poliinsaturados possuem inúmeros efeitos contra as células malignas e o seu emprego em seres humanos no combate ao câncer está muito perto, pois em modelos animais e em cultura de células eles reduzem a proliferação celular, aumentam a apoptose, determinam a diferenciação celular, reduzem a neoangiogênese tumoral e reduzem 0 aparecimento de metástases. Existem evidências que quanto menor o conteúdo de AG intratumoral maior é o grau de malignidade do tumor. ${ }^{11}$

Uma das principais funções dos AGs n-3 e n-6 refere-se à sua conversão enzimática em eicosanoides. Os eicosanoides possuem inúmeras atividades biológicas, pois são capazes de modular a resposta inflamatória e a resposta imunológica; além de influenciar na agregação plaquetária, no crescimento e na diferenciação celular. A produção de eicosanoides inicia com a liberação dos AGs poliinsaturados da membrana fosfolipídica pela ação de várias fosfolipases. Liberados da membrana, esses AGs servem como substratos para cicloxigenases, lipoxigenases e citocromo P450 monoxigenase. ${ }^{12}$

A presença de micronúcleos na mucosa bucal pode ser um preditor do risco de câncer nas vias aerodigestivas superiores, inclusive nas fases pré- 
malignas, como a leucoplasia oral. Estudo encontrou maior frequência de células bucais com micronúcleos em pacientes com câncer de mama e de útero e seus parentes de primeiro grau, em relação aos grupos controles. ${ }^{13}$

De acordo com alguns autores, os micronúcleos são massas de cromatina que são moldados como núcleos pequenos e aparecem perto do núcleo nas células em interfase. ${ }^{9,12-13}$ A prevalência de dano ao DNA é também influenciada por fatores relacionados ao estilo de vida, fumar, beber álcool, trabalhando longas horas, não dormir o suficiente, inatividade física, tipo de dieta e estresse psicológico contribuem para um aumento da prevalência e consequente desenvolvimento de doenças, incluindo câncer. ${ }^{10}$

O sobrepeso e a obesidade contribuem fortemente para a carga de doenças crônicas e de incapacidades, que vão desde dificuldades respiratórias até condições graves, como doença coronariana e certos tipos de câncer. Estima-se que $90 \mathrm{mil}$ mortes por câncer poderiam ser evitadas a cada ano, se a população adulta mantivesse um peso corporal adequado e que, aproximadamente, 20-33\% dos casos de câncer mais comuns possam ser atribuídos ao peso corporal excedente e à inatividade física. ${ }^{8}$

Além disso, a obesidade está descrita como associada ao aumento no risco do câncer colorretal, sendo a prevalência mais nítida no sexo masculino. Questiona-se, no entanto, como fator mais importante, não a obesidade em si, quantificada pelo Índice de Massa Corporal (IMC), mas a presença da obesidade visceral, melhor identificada pela distribuição andróide da gordura, podendo ser indiretamente estudada pela medida da circunferência abdominal, ou melhor, ainda, pela razão cintura quadril. ${ }^{14}$

Embora os estudos tenham demonstrado que o excesso de peso aumenta o risco de câncer do cólon, rim, esôfago, endométrio e de mama (este em mulheres pós-menopausa), dando suporte à ideia de que o controle da obesidade pode representar uma importante medida de prevenção de muitos tipos de câncer na sociedade moderna..$^{7,10}$

0 consumo de ômega- 3 como forma de redução da incidência de doenças cardiovasculares e câncer

No início do século $X X, a$ importância dos AG era relacionada exclusivamente a sua maneira eficaz de armazenar energia, que podem ser sintetizadas pelo organismo a partir de proteínas e carboidratos. ${ }^{15} \mathrm{~A}$ partir de então, inúmeras evidências asseguram que dieta pobre em AG é relacionada às síndromes que em algumas circunstâncias podem resultar em morte. A maior parte dos conhecimentos sobre os efeitos benéficos do óleo de peixe para a saúde foi proveniente de estudo epidemiológico em populações que têm dietas ricas em ômega-3. ${ }^{10}$

Os AGs $n-3$ podem reduzir o crescimento dos tumores de mama estrógeno dependentes pela redução da estimulação estrogênica nesses tumores. ${ }^{7} 0$ nível de DHA no tecido adiposo das mamas de pacientes que 
 \\ ANOHEALTH \\ apresentaram \\ remissão \\ total ou} parcial, em resposta ao tratamento com drogas citotóxicas, foi maior que em pacientes que não responderam ao tratamento ou que apresentaram progressão da doença. ${ }^{16}$

Em animais, o consumo de AGs ômega-3 reduz a proliferação maligna de células cancerosas humanas neles implantadas, aumenta a eficácia da quimioterapia, diminui os efeitos colaterais da quimioterapia e diminui os efeitos mórbidos do câncer. Esses AG são importantes em vários aspectos da saúde, incluindo a cardiovascular. ${ }^{11}$

Os benefícios do consumo dietético do ômega-3 já foram comprovados na prevenção e estabilização de diversas doenças e condições patológicas, inclusive no câncer. ${ }^{12}$ Suplementos de ômega-3 estão sendo amplamente investigados através de dietas enriquecidas quanto ao seu efeito na evolução clínica e os resultados em pacientes com câncer, principalmente na modulação da resposta inflamatória e imunológica e na melhora da síndrome da caquexia relacionada ao câncer, contudo, ainda não foram estabelecidas recomendações específicas para sua ingestão no que diz respeito à referida patologia. ${ }^{14,17}$

A obesidade gera um estado de inflamação crônica, de baixo grau caracterizado pela secreção excessiva de mediadores inflamatórios por adipócitos, macrófagos e outras células, incluindo a microbiota intestinal. Estes fatores próinflamatórias perturbam a homeostase metabólica e, assim, promove a resistência à insulina, diabetes tipo 2 , doença cardiovascular e câncer. ${ }^{9}$
ISSN 2236 - 1987

Numerosos

epidemiológicos têm demonstrado efeitos benéficos à saúde com o uso de ômega-3 AGs poli-insaturados, incluindo reduções de inflamação, hiperlipidemia e melhorou a sinalização de insulina. ${ }^{10}$ As propriedades anti-inflamatórias e metabólicas de reprogramação de ômega-3 têm sido mostradas para retardar o aparecimento de câncer em diversos modelos animais, anulando os efeitos pró-tumorgênicas da obesidade. Em ofertas diferenciadas, já foram atribuídas a ele qualidade farmacológica para amenizar inflamações, ativação citoquímica e hipermetabolismo. Agindo como moduladores da resposta nutricional e metabólica no câncer avançado, combatendo a caquexia e melhorando a evolução clínica e qualidade de vida dos enfermos. ${ }^{14-15,17}$

\section{CONSIDERAÇÕES FINAIS}

Portanto, fica evidente que os resultados obtidos em alguns trabalhos possuem linhas de pesquisa comuns entre si, foram criadas duas categorias, a fim de facilitar a discussão entre os autores, sendo possível observar à importância do ômega-3 na redução de micronúcleos em células da mucosa oral em pacientes com obesidade. Observou-se que AGs poli-insaturados possuem inúmeros efeitos contra as células malignas e o seu emprego em seres humanos no combate ao câncer está muito perto, pois em modelos animais e em cultura de células eles reduzem a proliferação celular, aumentam a apoptose, determinam a diferenciação 
celular, reduzem a neoangiogênese tumoral e reduzem o aparecimento de metástases.

0 consumo de ômega- 3 reduz a incidência de doenças cardiovasculares e câncer devido aos AGs n-3 reduzirem o crescimento de tumores cancerígenos e a proliferação maligna de células cancerosas humanas nele implantadas. Em relação a quimioterapia, o consumo de ômega-3 diminui os feitos colaterais e os efeitos mórbidos do câncer. Assim, esses AGs são importantes em vários aspectos da saúde, como os supracitados, bem como para o sistema cardiovascular.

\section{REFERÊNCIAS}

1 Luzhna L, Kathiria P, Kovalchuk 0. Micronuclei in genotoxicity assessment: from genetics to epigenetics and beyond. Frontiers in genetics [Internet]. 2013[cited 2019 Jun 30];4(131):1-17. Available from: https: //www.ncbi.nlm.nih.gov/pmc/a rticles/PMC3708156/pdf/fgene-0400131.pdf

2 Pedersoli AGA, Gonçalves RCC, Oliveira LML de, Correia JSC. Ômega-3 e redução dos triglicerídeos no paciente com doença cardiovascular. Revista saber científico [Internet]. 2015[acesso em 2019 jun 30];4(1):4651. Disponível em: http: / / revista.saolucas.edu.br/index. $\mathrm{php} / \mathrm{resc} /$ article/view/527/PDF

3 Chan EJ, Cho L. What can we expect from omega-3 fatty acids? Clevel. clin. j. med. [Internet]. 2009[cited 2019 Jun 30];76(4):245-51. Available from: https://mdedge-files-live.s3.us-east2.amazonaws.com/files/s3fs- public/issues/articles/media_e5e9d4a _245.pdf

4 Thomas P, Holland N, Bolognesi C, Kirsch-Volders M, Bonassi S, Zeiger, et al. Buccal micronucleus cytome assay. Nat. Protoc [Internet]. 2009[cited 2020 May 26];4(6):825-37. Available from: https://www.nature.com/articles/npr ot. 2009.53

5 Gonzalez SEF, Anguiano EA, Herrera AM, Escutia-Calzada D, Pichardo CO. Cytotoxic, pro-apoptotic, pro-oxidant, and non-genotoxic activities of a novel copper (II) complex against human cervical câncer. Toxicology. [Internet]. 2013[cited 2020 May 26];314(1):155-65. Available from: https://www.sciencedirect.com/scien ce/article/abs/pii/S0300483X1300237 0?via\%3Dihub

6 Samanta S, Dey P, Nijhawan R. Micronucleus in cervical intraepithelial lesions and carcinoma. Acta cytol. [Internet]. 2011[cited 2020 May 26];55(1):42-7. Available from: https://www.karger.com/Article/Pur chase/320792

7 Skulas-Ray AC, Kris-Etherton PM, Harris WS, Heuvel JPV, Wagner PR, West SG. Dose-response effects of omega-3 fatty acids on triglycerides, inflammation, and endothelial function in healthy persons with moderate hypertriglyceridemia. Am. j. clin. nutr. [Internet]. 2011[cited 2019 Jun 30];93(2):243-52. Available from: https://academic.oup.com/ajcn/artic le/93/2/243/4597603

8 Sears DA, Udden MM. Howell-Jolly bodies: a brief historical review. Am. j. med. sci. [Internet]. 2012[cited 2020 May 26];343(5):407-9. Available from: https://www.sciencedirect.com/scien 


\section{, \\ NURSING \\ AND HEALTH \\ ce/article/abs/pii/S000296291530973 3}

9 Melo AST,Cândido APC. Avaliação dos aspectos nutricionais, das condições de saúde e do perfil sociodemográfico de idosos atendidos no departamento de saúde do idoso - Juiz de Fora, MG. Rev. APS. [Internet]. 2016[acesso em 2019 jun 30];9(4):533-45. Disponível em: https://periodicos.ufjf.br/index.php/ aps/article/download/15827/8256

10 Simão ANC, Godeny P, Lozovoy MAB, Dichi JB, Dichi I. Efeito dos ácidos graxos $n-3$ no perfil glicêmico e lipídico, no estresse oxidativo e na capacidade antioxidante total de pacientes com síndrome metabólica. Arq. bras. endocrinol. metab. [Internet]. 2010[acesso em 2019 jun 30];54(5):463-9. Disponível em: http://www.scielo.br/pdf/abem/v54n 5/06.pdf

11 Felippe Júnior $\mathrm{J}$ de. Óleo de peixe ômega-3 e câncer: diminuição da proliferação celular maligna, aumento do apoptose, indução da diferenciação celular e diminuição da neoangiogênese tumoral. Medicina complementar [Internet]. 2011[acesso em 2019 jun 30]. Disponível em: http://www.medicinabiomolecular.co $\mathrm{m} . \mathrm{br} / \mathrm{biblioteca/pdfs/Cancer/ca-}$

0379.pdf

12 Cerqueira SRP. Os ácidos gordos ómega-3 e os seus efeitos antiinflamatórios [dissertação] [Internet]. Porto (PT): Universidade Fernando Pessoa Faculdade de Ciências da Saúde Departamento de Ciências Farmacêuticas FCS (DCF);2013[acesso em 2020 maio 26]. Disponível em: https://bdigital.ufp.pt/bitstream/102 84/4162/1/Os\%20ácidos\%20gordos\%20
ISSN 2236 - 1987

ómega-

3\%20e\%20os\%20seus\%20efeitos\%20antiinflamatórios.pdf

13 Sousa RMR P, Sobral DP, Paz SMRS da, Martins MCC do. Prevalência de sobrepeso e obesidade entre funcionários plantonistas de unidades de saúde de Teresina-PI. Rev. nutr. PUCCAMP. [Internet]. 2007[acesso em 2019 jun 30];20(5):473-82. Disponível em:

http://www.scielo.br/pdf/rn/v20n5/ a03v20n5.pdf

14 Souza EB de, Moreira A, Benedetti NCM, Saron MLG, Neves A. dos S. A influência do estado nutricional e da ingestão alimentar na aprendizagem escolar. Cadernos unifoa [Internet]. 2015[acesso em 2019 jun 30];10(29):105-13. Disponível em: http://revistas.unifoa.edu.br/index.p $\mathrm{hp} /$ cadernos/article/view/370/401

15 Malta DC, Andrade SC, Claro RM, Bernal RTI, Monteiro CA. Trends in prevalence of overweight and obesity in adults in 26 Brazilian state capitals and the Federal District from 2006 to 2012. Rev. bras. epidemiol. [Internet]. 2014[cited 2019 Jun 30];17 Suppl.1:267-76. Available from: http: / / www.scielo.br/pdf/rbepid/v17 s1/1415-790X-rbepid-17-s1-00267.pdf

16 Lobo ACM, Velasque LFL. Revisão de literatura sobre os efeitos terapêuticos do açaí e sua importância na alimentação. Biosaúde [Internet]. 2016[acesso em 2019 jun 30];18(2):97106. Disponível em: http://www.uel.br/revistas/uel/inde x.php/biosaude/article/view/27624/2 0446

17 Siddiqui RA, Harvey KA, Zaloga GP, Stillwell W. Modulation of lipid rafts by 
omega-3 fatty acids in inflammation and cancer: implications for use of lipids during nutritional support. Nutrition in clinical practice. [Internet]. 2007[cited 2020 May 26];22(1):74-88. Available from: https: / /onlinelibrary.wiley.com/doi/a bs $/ 10.1177 / 011542650702200174$ 